\title{
The availability of the basic oral health care package in the Western Cape
}

SADJ July 2017, Vol 72 no 6 p258 - p261

DA Smit', YI Osman²

\begin{abstract}
The majority of the uninsured population in the Western Cape, estimated at about 4.5 million, is dependent on public dental clinics. Dental caries remains the most common chronic disease world-wide. Caries experience among 6-year-old children in the province increased from $82 \%$ to $84 \%$ in the period $2002-2015$. Oral health programmes to promote good oral health at schools and clinics are very limited. A cross-sectional study investigated 128 dental clinics in the Western Cape to determine the availability of the basic oral health care package, which includes promotion of oral health, prevention of oral disease and basic oral health treatment. Less than a third (31.5\%) offered the basic treatment package and slightly less than two thirds (65\%) were offering only dental extractions. Despite clear policy guidelines, public dental care delivery in Western Cape is seen to be not adherent to the guidelines, norms and standards. There is an unavailability of dentists outside the main clinics and it appears there is a minimal focus on prevention of oral disease and promotion of good oral health. Dental caries must be recognized as an epidemic in the Western Cape, and basic oral health care should be made available.
\end{abstract}

\section{BACKGROUND}

About 4.5 million persons in the Western Cape have no dental insurance and must rely on public dental clinics simply because they do not have the financial means to consult a private dentist. There are approximately 120 public dental clinics in the Western Cape at which it may be expected that a basic oral health package should be offered. This service would include promotion of oral health, preventing oral disease and basic oral health treatment including an oral examination, bitewing radiographs, simple 1-3-surface restorations, and dental extractions. ${ }^{1-3}$ The majority of the clinics may not sufficiently equipped to offer even that basic package. ${ }^{1}$

Nevertheless, several good policy documents point the way for improved oral health delivery in the public sector.

1. DA Smit: BChD (Stellenbosch/Western Cape), MChD (CommDent) (Western Cape). Department of Maxillo-Facial- and Oral Surgery University of the Western Cape.

2. YI Osman: $B C h D, M C h D$ Western Cape), BBA (Hons), MBA (Stellenbosch), PGD HM (Cape Town). Dean, University of the Western Cape.

\section{Corresponding author}

DA Smit:

Department of Maxillo-Facial- and Oral Surgery, University of the

Western Cape. E-mail: dirkiesmit@gmail.com
These include the Norms and Standards for Oral Health Care in SA, ${ }^{2}$ National Oral Health Strategy ${ }^{3}$ and the National Oral Health Policy. ${ }^{4}$

\section{INTRODUCTION}

Dental caries remains worldwide the most common chronic disease. ${ }^{5}$ According to the last National Children Oral Health Survey conducted in 1999 - 2002 the caries experience among 6-year-olds stood at $82.3 \%{ }^{6}$ This figure was much higher than the National Mean of $50 \%$. Recent data from the Oral Health Service in the Province indicates that the caries experience amongst this age group has increased to $84 \% .^{7}$

It is a priority for a dentist in the public sector to attend to patients with pain and sepsis. ${ }^{3}$ These patients are finally driven to seek help, and at that stage, the most feasible treatment option is to extract the tooth in order to relieve the pain. ${ }^{8}$ There are many pre-school children who suffer from tooth ache associated with dental caries and in some instances they are treated under general anaesthetic. A study conducted in the Western Cape by Peerbhay and Barrie (2012) showed that only $0.0001 \%$ of all treatment done in the public sector on pre-school patients under general anaesthesia were for dental restorations while 99.94\% were for extractions. The mean number of teeth being extracted per patient was ten. ${ }^{8}$

Children with poor oral health are 2.3 times more likely to perform poorly in $\mathrm{schoo}^{9}$ and yet programmes to promote good oral health in the school setting and at clinics are very limited. Indeed, there is an uncertainty regarding the degree of availability of these services in the public sector. This paper reports on a study conducted in 2014 which aimed at establishing the availability of the basic oral health care package in the Western Cape.

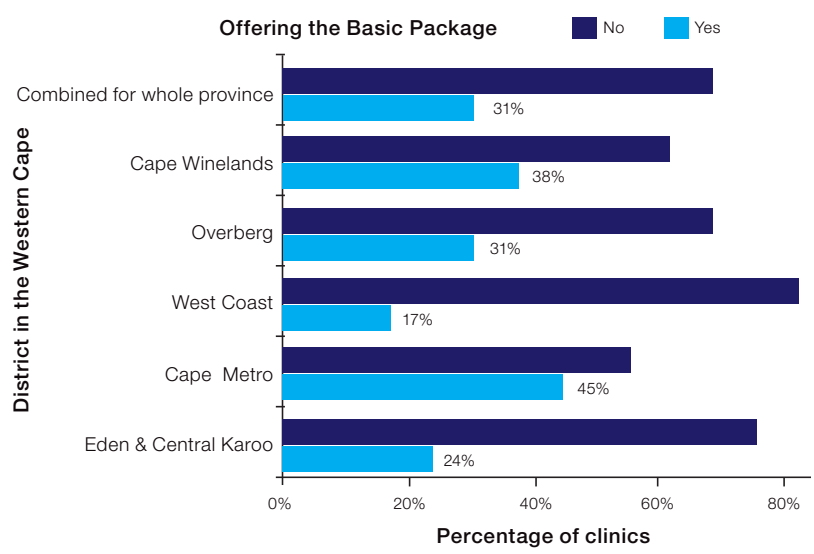

Figure 1: Availability of the basic oral health care package 


\section{MATERIAL AND METHODS}

A cross-sectional study was conducted to describe the availability of the basic oral care package in the Western Cape. The study included 128 dental clinics $193 \%$ of all clinics) and interviews were conducted with 60 dentists, that is $88 \%$ of all dentists employed by Government of the Western Cape. Available National and Provincial policy guidelines were used as a reference point to determine which procedures should be included in the basic package. Telephones were used to administer a questionnaire in the collection of data. The most recent census data ${ }^{10}$ was used to calculate dentist/population ratios.

\section{Aim}

To determine the availability of basic oral health care offered through the Provincial Dental Clinics in the Western Cape.

\section{Objectives}

- To determine the proportion of clinics that offer the basic oral health care package

- To calculate oral health professional : population ratio for each district

- To determine the number of days per month the clinic is visited by a dentist

- To ascertain work time distribution of dentists

- To investigate oral health promotional programmes and oral disease prevention activities offered in the public sector

- To investigate reasons for difficulties in delivery of services.

\section{Data analysis}

Data was recorded on a customized data capture sheet on Microsoft Word $2010^{\circledR}$ and the statistical analysis was completed on Microsoft Excel $2010^{\circledR}$ and Epi Info. ${ }^{7}$ A variety of statistical tests were used e.g. Student's t-test for means and Chi-square test for proportions. Statistical level of significance was determined as $p<0.05$.

\section{Ethical considerations}

- The research proposal was approved by the Senate and Faculty Research Ethics Committee of the University of the Western Cape (13/3/4) as well as by the Research Ethics Committee of the Government of the Western Cape (RP 042/2014).

- Participation was on a voluntary basis.

- The identity of the participants remained anonymous at all times.

- A signed informed consent form was obtained from all participants.

\section{RESULTS}

The proportion of clinics offering the basic oral health treatment package

Less than a third (31.5\%) of all clinics offered the basic treatment package (Figure 1) and just under two thirds (65\%) were offering dental extractions only. Less than half (43\%) were able to offer a scale \& polish and/or fillings while bitewing radiographs could be taken at only $30 \%$ of clinics. Fissure sealants which are essential to dental caries prevention were done at only $37 \%$ of clinics. The Metro health district had the best record in the Province with almost half of the clinics being able to offer the basic package compared with only $17 \%$ in the West Coast district.

There was an association between the ability to offer the basic package and the clinic type $(p<0.005)$. Main clinics were defined as a facility at which the dentist was based and the presence of the dentist could be guaranteed at

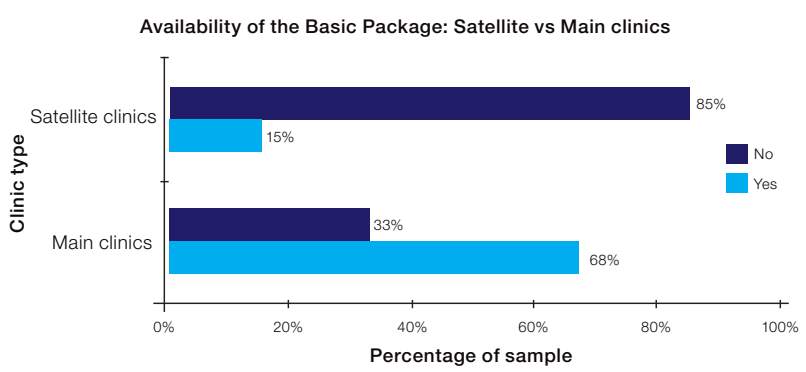

Figure 2: Availability of the basic oral health care package: Satellite vs Main clinics

least for a portion of the day. Satellite clinics were usually more remote and visited infrequently by a dentist; once a week, twice a week or on a monthly basis. Main clinics were 4.5 times more likely to offer the basic package compared with Satellite clinics (Risk Ratio $=4.5$ ). Only $15 \%$ of Satellite clinics could offer the basic package compared with $68 \%$ of Main clinics (Figure 2).

There was an association between the ability to offer the basic package and the geographic location of the clinic $(p<0.009)$. Metro clinics were located in the Metro Health District which resembles the City of Cape Town. Rural clinics were defined as clinics situated outside the Metro Health District. Metro clinics were 2.5 times more likely to offer the basic package compared with Satellite clinics (Risk Ratio $=4.5$ ). Only a quarter of Rural clinics could offer the basic package while at least half of all the Metro clinics could deliver those services (Figure 3).

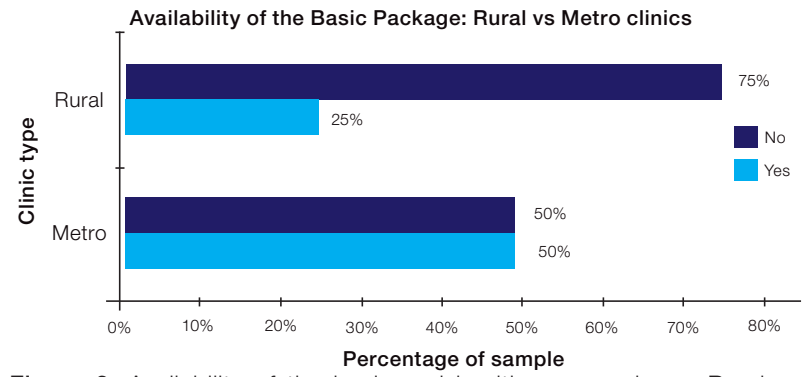

Figure 3: Availability of the basic oral health care package: Rural vs Metro clinics

\section{Dentist : population ratio}

In the present study, the dentist : population ratio for the Western Cape was calculated for each district (Table 1). The ratio for the whole province was 1: 71875 (Table 1). The two districts having the highest proportions of population were Metro $(1: 80,429)$ and Eden $(1: 72,069)$. These ratios are all far from the recommended ratios as stipulated in the National Oral Health Strategy (2005), that document recommending figures of $1: 60,000$. The current study found that there is a shortage of 13 dentists, 18 Oral Hygienists and 43 dental assistants in the Province.

\begin{tabular}{|l|c|c|c|}
\hline Table 1: Dentist : Population ratios in the Western Cape \\
\hline District & Dentists & $\begin{array}{c}\text { Uninsured } \\
\text { population }\end{array}$ & $\begin{array}{c}\text { Dentist : } \\
\text { Population ratio }\end{array}$ \\
\hline Metro & 38 & 3056296 & $1: 80429$ \\
\hline West Coast & 5 & 263385 & $1: 52677$ \\
\hline Cape Winelands & 10 & 595646 & $1: 59565$ \\
\hline Overberg & 4 & 198613 & $1: 49653$ \\
\hline Eden & 7 & 504483 & $1: 72069$ \\
\hline Central Karoo & 1 & 53422 & $1: 53422$ \\
\hline Total & 65 & 4671844 & $1: 71875$ \\
\hline
\end{tabular}




\section{Availability of dentists at public clinics}

The availability of dentists at various clinics was ascertained by recording the number of days in a month that a dentist was physically present at the clinic (a maximum of 20 days). The mean number of days that a dentist is present overall at clinics is 7.3 days $(S D=7.8)$ per month. There was a significant difference between professional attendances at Main and Satellite clinics $(p<0.0005)$. At Main clinics, a dentist was present for 16.6 days compared with Satellite clinics where dentists were present for only 3.2 days per month.

\section{Working time distribution}

Working time was measured by the number of hours in a normal working day (8 hours/100\%) that the dentist rendered services at the relevant Main clinic (where the dentist was based). Working time was further categorized into performing dental extractions, fillings, other clinical services, administrative duties, or driving to nearby schools or clinics.

The mean time per day which was dedicated to dental restorations was 2.06 hours which represented $26 \%$ of a working day and in this instance there was no significant difference between Metro and Rural clinics. Half of the time (3.78 hours) in a working day was dedicated to performing dental extractions and there was a significant difference between Metro and Rural clinics ( $p<0.05)$. Dentists from districts dedicated a larger proportion of the day to extractions compared with the Metro clinics. The mean time per day allocated to administrative duties was 1.34 hours and there was no significant difference in this aspect of work between dentists from Metro and Rural clinics. The mean time per week which was taken up in driving to surrounding clinics and schools was 1.8 hours and there was a significant difference between dentists from Metro and Rural clinics $(p<0.0005)$. Dentists who were working in the Rural districts spent more time driving to schools and Satellite clinics compared with dentists in the Metro clinics.

\section{Oral promotion \& prevention}

Almost two thirds of the responding dentists visited schools at least once a year to render oral health services which were mostly dental screening. Only $20 \%$ of dentists visited baby clinics annually while $15 \%$ visited crèches (Figure 4). The "Road to Health" chart/booklet for infants and young children is a national instrument which is used to monitor growth and development of an infant and young child. On certain days a special clinic (baby clinic) is scheduled for infants and toddlers to monitor their growth and development. Page 20 in the booklet is dedicated to oral health and can be completed by a dentist, dental therapist or an oral hygienist. The majority of dentists have knowledge of the oral health page but only $47 \%$ actually completed it at least once per year.

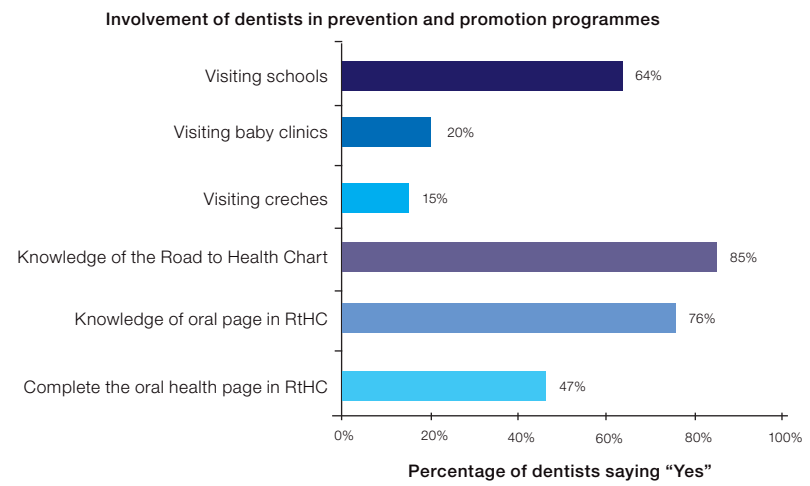

Figure 4: Proportion of dentists who visit schools, baby clinics and crèches as well as provide information on the Road to Health Chart
Factors contributing to unavailability of the basic package Whilst the majority of dentists do have access to a government vehicle to use in visits to Satellite clinics and surrounding crèches, these are not always readily available.

The time required to repair or replace dental equipment is an essential component in the consistent provision of the basic oral health care package. The mean equipment repair time was three weeks and the mean time of acquiring new dental equipment was seven months. There was no significant difference between metro and rural clinics regarding equipment replacement/repairing times ( $p>0.05$ ).

Waiting times for appointments for dental treatment under general anesthesia, dental restorations and general waiting time for patients at the dental clinic were also included in the study. The mean waiting time for a dental patient from arrival at the clinic until contact with a clinician was 1.5 hours. The waiting time for an appointment for treatment under general anesthesia (GA) for a child was three months. The mean waiting time for an appointment for dental fillings was three weeks.

According to the study, dentists indicated that the basic package could not be offered at clinics for various reasons. A major obstacle was the unavailability of equipment including dental chair light, suction, dental material, film and radiograph developer. A shortage of oral health staff and poor working environments were further problems, given that at some clinics dentists were working on a plastic or ordinary desk chair and sharing a surgery with other professionals, resulting in a lack of privacy.

The most common main complaint of patients was pain and sepsis, resulting in the majority of treatments being dental extractions. However, some participants also mentioned that the community demanded extractions instead of restorations.

Time constraints due to high numbers of patients or extensive travel distances also played some role in limiting the variety of dental care which could be offered.

\section{DISCUSSION}

Activities involving oral health promotion and prevention of oral disease are essential components of basic oral health care as stipulated by National Oral Health Policy documents.

A comprehensive oral health plan had been approved by the Provincial Minister in 2007. In this planning document, specific recommendations for basic oral health care were made in terms of instrumentation, equipment and infrastructure requirements for public dental care. As yet, it has not been implemented due to limited resources.' In March, 2014, a policy document, "Healthcare 2030 - The Road to Wellness", was issued by the Provincial Government of the Western Cape. ${ }^{11}$ The Oral Health chapter in this document alludes to the importance of oral conditions, the oral health services platform which require strengthening, a focus shift from curative services towards preventive and promotion services and the role of the Oral Health Centre in the provision of outreach and support to District Health services. There is also mention of the community-based services designed to support Integrated School Health Programmes and home-based care services. ${ }^{12}$ These platforms should be used to drive supervised tooth brushing strategies, in conjunction with services from the Department of Basic Education as well as Social Development.

The present study also showed that the line of reporting from dentists to their managers also seemed problematic. 
Participants indicated that they report to the Primary Health Care Practitioner of a health sub-district. This person is usually a nurse who is not familiar with oral health procedures and the scope of clinical practice of the different oral health professionals. Procurement for dental instrumentation and equipment is channeled through this officer and often dentists experience frustration as the requirements and standards of a public dental clinic are not always appreciated. Dental statistics and work load requirements are also managed by the Primary Health Care Practitioner. This often leads to further frustration among dentists when headcounts and the number of extractions are regarded as the main indicators for the delivery of oral health services. There is considerable scientific evidence that these statistics are inaccurate indicators for measuring the quality of oral health services. ${ }^{13}$ Logically, oral health services should surely be managed by an oral health professional.

The dentist : population ratio in South Africa is extremely low compared with other middle income countries. ${ }^{14}$ The National Policy for Oral Health in South Africa suggested that the dentist : population ratio should be 1: 15,000, as calculated from the National Oral Health Survey 1988/1989.14 That ratio has changed dramatically to a suggestion that there should be one dentist for 60000 people. ${ }^{2}$ According to the most recent National Policy documents the oral health professional: population ratios should be as follows:

- Oral Hygienist: Population 1: 100000

- Dental Therapist: Population 1: 60000

- Dentist: Dental Therapist 1: 5

- Dental Therapist: Oral Hygienist $5: 1$

These ratios are questionable since no scientific evidence justified the recommended ratios. The question should also be asked whether these ratios are still applicable to the public sector today, recognising the effects of urbanization and the rising prevalence of dental caries. Ratios of oral health clinician: population should preferably be calculated through utilising the needs/ services and demand-based approach. These calculations are depended on DMFTscores which are specific for different age groups..$^{15}$

A serious case can be made for the need to appoint a specialist in Community Dentistry to assist the Provincial Government with policy development, economic evaluation, epidemiological surveillance, statistical analyses and the management of oral health services. The lack of specialist services outside the Oral Health Centre is contributing to escalating waiting lists, the inability to apply epidemiological and statistical trends and the escalating burden of oral disease in the Western Cape. Other dental specialist groups such as Orthodontics, Oral Pathology, Prosthodontics, Periodontics and Maxillo-Facial and Oral Surgery are also restricted to the Oral Health Centre. These specialists have a main focus on tertiary clinical services, research and post-graduate teaching and learning. Consideration should therefore be given to the appointment of dental specialists at selected regional and district hospitals to enable the provision of equitable oral health specialist care to public patients.

\section{CONCLUSION}

The majority of public dental clinics in the Western Cape are unable to offer basic oral health care and about two thirds of clinics restrict services to extractions. The most recent Provincial Policy document, "The Road to Wellness" and the National Oral Health Strategy document describe the picture of oral health care for the future as well as proposing the structure and system to address inequities in providing for oral health. However despite these clear policy guidelines, public dental care in Western Cape is found to be not adhering to these norms and standards. The socioeconomic and psychological factors of oral disease are also being ignored. Infrequent visits at Satellite clinics by dentists emphasize unavailability of professionals outside the Main clinics. Infrequent visits to schools and crèches and the poor completion rate of the oral health page in the Road to Health booklet highlight a minimal focus on oral disease prevention and on the promotion of good oral health.

Basic Oral health should be regarded as a human right deserved by each South African, not only the $16 \%$ of the population who have access to private health care. Managers and decision makers should implement existing health strategies to prevent oral disease and promote good oral health.

The availability of basic oral care is essential, exemplified by the rising prevalence of dental caries amongst children. Furthermore, it is time that dental caries must be recognized as an epidemic in the Western Cape. It is a disease affecting the majority of society and it restricts the quality of life. Recognition of oral health issues and making basic oral health care generally available is essential to address the oral health problems in the Western Cape.

\section{References}

1. Provincial Government of the Western Cape. Department of Health, Comprehensive Oral Health Service Plan for the Implementation of Healthcare 2010, Final Draft, December 2007.

2. Department of Health. Norms and Standards for Oral Health in South Africa, 2005; Available from <http://www.doh.gov.za/ docs/policy/strategy.pdf > Accessed on the 4th of October 2012

3. Department of Health. South African National Oral Health Strategy, 2004; Pretoria, 1 - 31.

4. Government of the Western Cape. National Oral Health Policy, 2003; Available from https://www.westerncape.gov.za/ text/2003/national_policy_oral_health_sa.pdf. Accessed on 2 February 2016.

5. Selwitz, RH, Ismail, Al, Pitts, NB. Dental caries. The Lancet 2007; 369 (9555):.51-9.

6. Van Wyk, PJ. Caries status and treatment needs in South Africa: Report of the 1999 - 2002 National Children's Oral Health Survey, South African Dental Journal 2004; 59 (6) 238 - 42

7. Smit, DA, Louw, AJ, Barrie, RB. The burden of dental caries in the Western Cape and providing an integrated oral health promotion approach , Unpublished article 2016.

8. Peerbhay, F, Barrie, RB. The burden of early childhood caries in the Western Cape Public Service in relation to dental general anaesthesia: implications for prevention, South African Dental Journal 2012; 67 (1): 14 - 9.

9. Blumenshine, S.L. Children's school performance: impact of general and oral health. Journal of Public Health Dentistry 2008; 68 (2): 82-7. Available at: http://www.ncbi.nlm.nih.gov/ pubmed/18221320.

10. Statistics South Africa. Census of South Africa, 2011, Available at: http://www.statssa.gov.za/?page_id=3955> Accessed on the 27th of Nov 2015.

11. Government of the Western Cape. Healthcare 2030. The Road to Wellness. Western Cape Government. Health. March 2014, Available at: https://www.westerncape.gov.za/.../health/ healthcare2030.pdf Accessed on 2 February 2016.

12. Shung King, M, Roper, M, Mabhena, N, Nel, A, Jones, S, Minnies, D, Swanepoel, D, Smit, D, Daviaud, E, Saloojee, H, Orgill, M, De Vries, P, English, R, Reid, S, Bothma, T. A strategic plan for the implementation of the Integrated School Health Programme, Department of Basic Education, Department of Health, Department of Social Development. 2014.

13. Barrie, RB. The Design, Implementation and Evaluation of a Management Information System for Public Dental Services. PhD thesis, University of the Western Cape, 2014.

14. Strachan, B, Zabow, T, Spuy, ZM. More doctors and dentists are needed in South Africa. South African Medical Journa 2011; 101: 523-8.

15. Kissoon-Singh, P. Planning oral health human resources for the province of KwaZulu-Natal, MSc Research Report (Community Dentistry), University of Pretoria, May 2001. 